\title{
Sperm tail axoneme alterations in the Wobbler mouse
}

\author{
J. E. Leestma and S. Sepsenwol*† \\ Departments of Pathology and *Physiology, Northwestern University School of Medicine, \\ 303 East Chicago Avenue, Chicago, Illinois 60611, U.S.A.
}

\begin{abstract}
Summary. In Wobbler mice (neurological mutants affected with progressive motor neurone degenerative disease) a defect in sperm tail mobility and axonemal geometry was observed. Similar but less extensive abnormalities were seen in the ciliary axonemes of the ductuli efferentes.
\end{abstract}

\section{Introduction}

The Wobbler mouse is an important animal model of motor neurone disease which results from a mutant autosomal recessive gene designated $w r$, first described by Falconer (1956) in a $\mathrm{C} 57 \mathrm{BL} / \mathrm{Fa}$ inbred mouse. Only animals homozygous for the trait show symptoms of disease which appears 3 weeks after birth and consists of weakness in the forelimbs and neck musculature, progressing to paralysis and contracture of forelimbs, a wobbly gait, and neurogenic muscular atrophy in affected muscle groups (Duchen, Falconer \& Stritch, 1966; Duchen, Stritch \& Falconer, 1968). Pathological studies have revealed a vacuolating degeneration of motor nerve cells in the spinal cord, brain stem, and portions of the brain. Heterozygous animals show no symptoms of the disease and can only be identified after breeding with a known heterozygote $(w r /+)$ producing Wobbler offspring $(w r / w r)$ (Duchen $e t$ al., 1968; Andrews \& Andrews, 1976). The condition shows many similarities to WerdnigHoffmann Disease and other hereditary forms of motor neurone disease in infants (Kohn, 1971).

Since the discovery of the Wobbler trait it has been necessary to perpetuate the gene by breeding heterozygous animals, since homozygotes (Wobblers) of either sex are unable to produce litters with each other or with normal mates (Duchen et al., 1968). The basis for this sterility has not been previously investigated. We undertook to evaluate causes for sterility in the male Wobbler.

\section{Materials and Methods}

The organs of 6 Wobbler males, 3-6 months old and weighing 20-25 g, were examined grossly and microscopically. Spermatozoa were removed from the vasa deferentia of 4 Wobbler mice and 4 control C57 mice and were examined under phase-contrast microscopy after dilution in Hanks balanced salt solution. Similar sperm suspensions were treated with 1-methyl-3-isobutyl xanthine, an agent known to enhance sperm motility (Chasin \& Harris, 1976), and were also assayed for adenylate cyclase activity (Braun, 1975).

Four Wobbler mice and 4 control mice were killed by intracardiac perfusion with $4 \%$ glutaraldehyde in phosphate buffer. Blocks of tissue from testis, ductuli efferentes, epididymis and vas deferens were examined ultrastructurally. The occurrence of axoneme alterations in Wobbler tissues was compared with that in control tissues.

† Present address: Department of Biology, University of Wisconsin at Stevens Point, Stevens Point, Wisconsin, 54481, U.S.A. 


\section{Results}

The Wobbler mice were about $25 \%$ smaller than age-matched control C57 mice. Apart from previously reported brain, neuromuscular and liver pathology (Andrews \& Andrews, 1976) we found no obvious gross or microscopic differences between controls and Wobblers. Sperm suspensions contained large numbers of spermatozoa, but those from the Wobblers contained only a very small number of motile cells. The immotile spermatozoa were normal in appearance with tails of normal length and form, and heads of normal appearance. Treatment with 1-methyl3-isobutyl xanthine clearly enhanced motility of the control but not of the Wobbler spermatozoa. Adenylate cyclase activity in Wobbler spermatozoa was not significantly different from that in the control spermatozoa. Ultrastructurally, the most striking abnormality observed in the Wobbler spermatozoa was the absence of from 1 to 4 outer doublets and their corresponding 'coarse' fibres. Most commonly these were doublets 4 through 7, and occasionally doublets 4 or 7 only (Pl. 1, Fig. 1). Less frequently supernumerary tubules were found, or central pairs were absent. When outer doublets were absent their 'spokes' were also usually absent. Some sperm tails showed vacuolation, increased density, or granularity, but most had intact plasma membranes. Abnormal axonemal complexes in sperm tails increased with distance from the testis where only about $5 \%$ of the spermatozoa were abnormal. In the ductus deferens nearly $70 \%$ of spermatozoa had altered geometry.

In the ciliated cells of the ductuli efferentes about $12 \%$ of ciliary axonemes from the Wobbler mice appeared abnormal while none in the controls showed alterations. The alterations were more subtle than those seen in spermatozoa (Pl. 1, Fig. 2); 1 or 2 outer doublets were missing or disarranged in or near doublet number 1 . Loss of one or more of the central tubules as well as occasional supernumerary tubules were also seen.

\section{Discussion}

Alterations in the fine structure of the axoneme have been noted rather infrequently in mammals. In man a systemic axonemal alteration has been reported in connection with the Kartagener's Syndrome (Kartagener, 1933) in which males are sterile and possess immotile spermatozoa; there is an homologous defect in sperm tails and respiratory cilia in which the 'side arms' (dynein) which normally join the outer doublets are lacking and the axonemes are immotile (Afzelius, Eliasson, Johnsen \& Lindholmer, 1975; Afzelius, 1976; Pedersen \& Mygind, 1976).

Similar alterations in the sperm tail axoneme have also been noted in house mice carrying a $t$ allele at the so-called $T$ locus (Olds, 1971), in subfertile bulls (Dag Defect) (Blom \& BirchAndersen, 1966), and in old spermatozoa undergoing degeneration in normal animals (Cooper \& Hamilton, 1977). These alterations consisted of deletion or extrusion of outer doublets 4-7 but were not always accompanied by infertility. Olson \& Linck (1977) have produced identical alterations in rat sperm tails by the addition of ATP to detergent solutions in which spermatozoa were suspended for chemical analysis. They observed that doublets 3-8, apparently firmly attached to the outer fibrous sheath of the principal piece, are held intact, while doublets 4-7 are

\section{PLATE 1}

Fig. 1. This group of sperm tails from the vas deferens of a Wobbler mouse reveals the most common alterations in geometry encountered: (A) lack of outer doublets 4-7 and with their spokes; (B) lack only of outer doublet 7 and a single spoke; (C) lack of doublets 4-7 but has greatly increased intertubular matrix as does a neighbouring sperm tail. Marker is $250 \mathrm{~nm}$.

Fig. 2. These cilia from a ductus efferens of a Wobbler mouse illustrate the subtle geometrical alterations encountered. The profiles with arrows illustrate some of the alterations seen. Marker is $250 \mathrm{~nm}$. 
PLATE 1

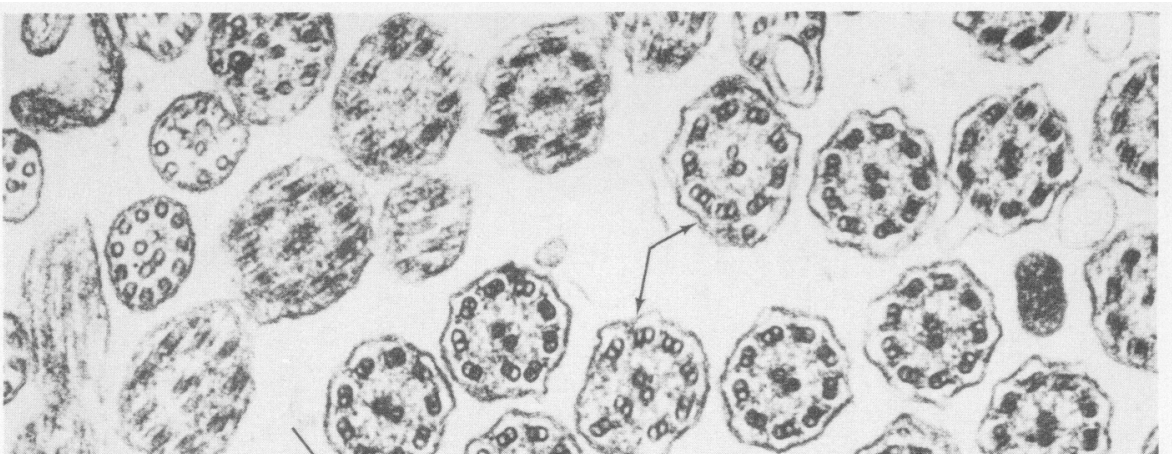

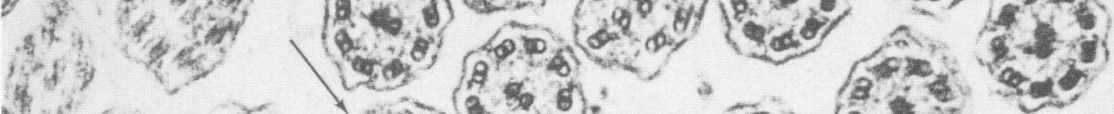

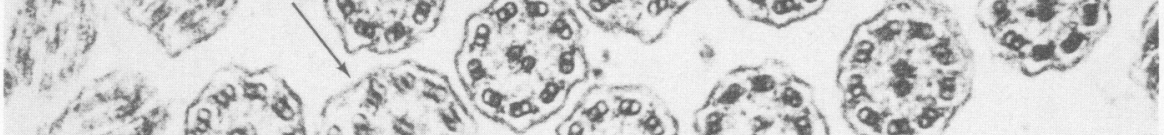

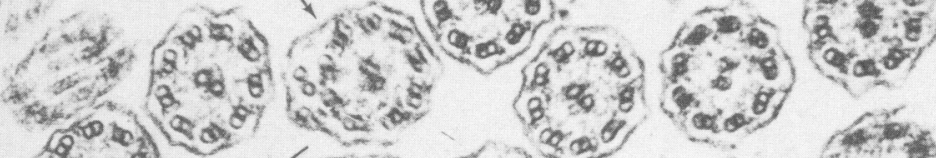

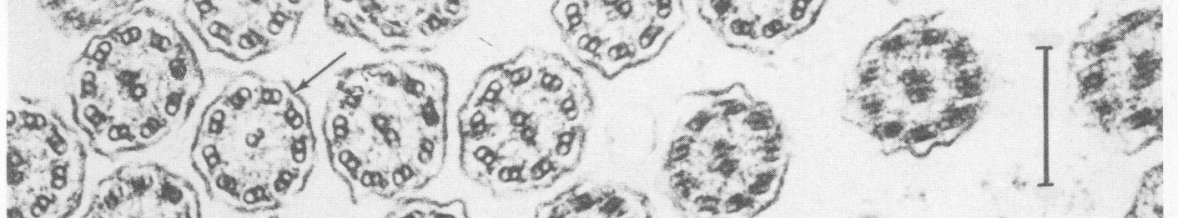

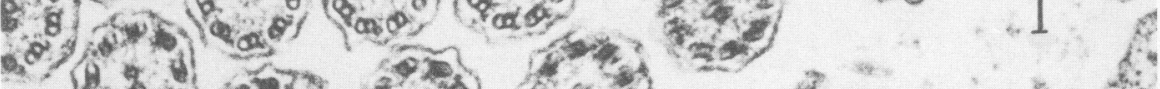

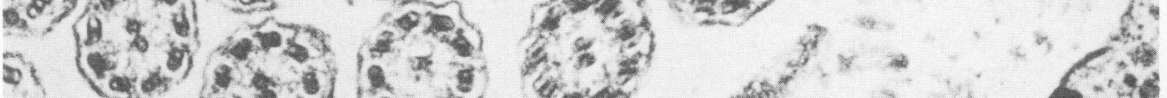

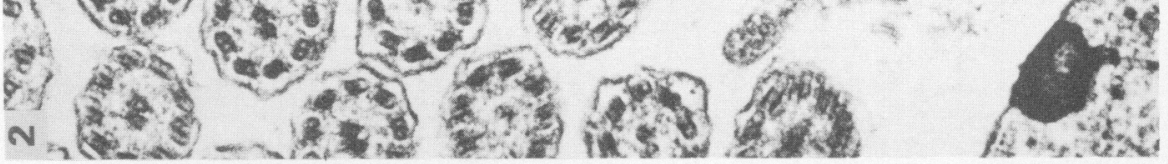

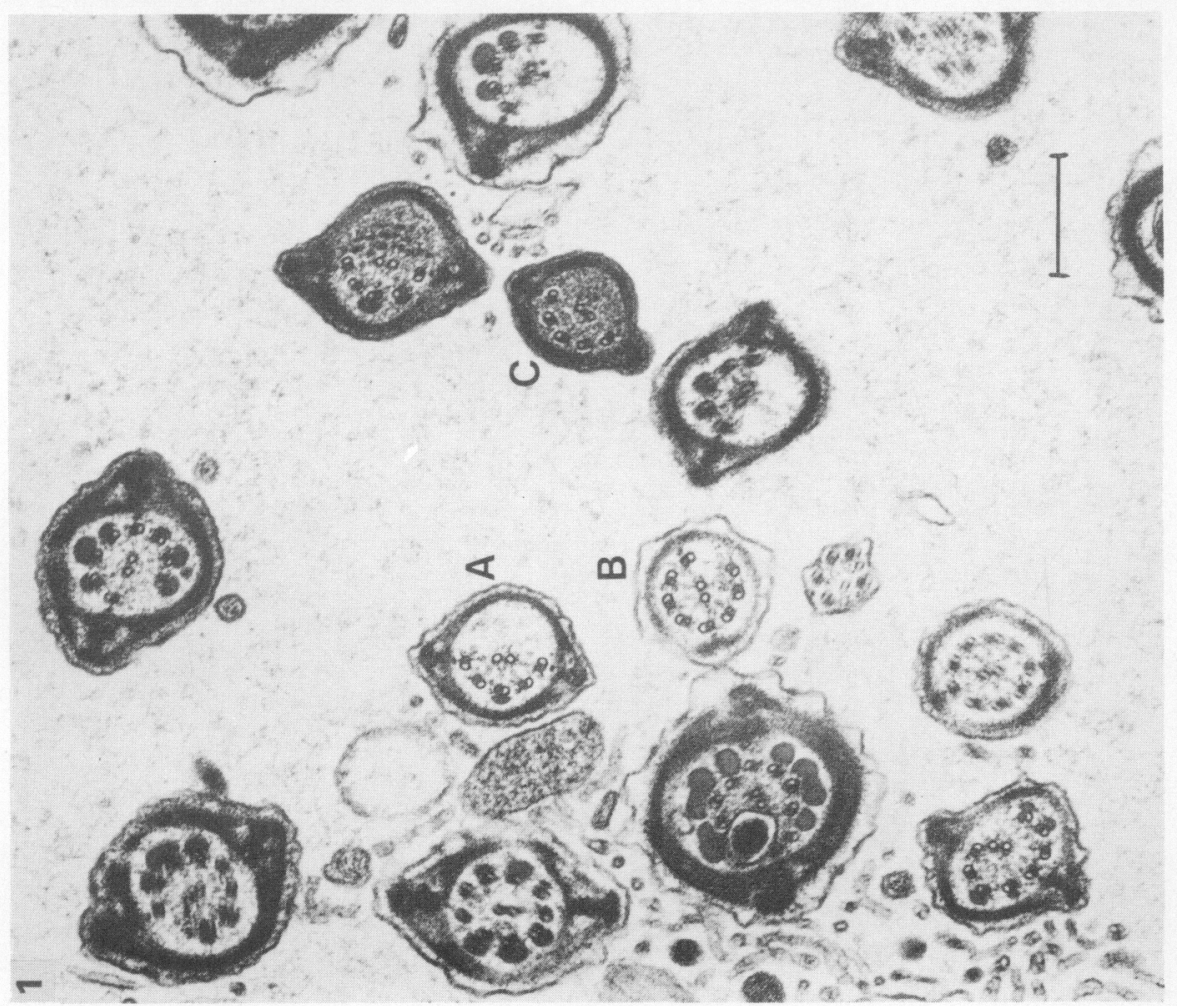

(Facing p. 268) 
extruded from the proximal portions of the sperm tail. Tails from which 4 doublets have been so extruded lose their motility and may retain the extruded portions of the axoneme as a flaccid, apparently extra tail. No such 'double' tails were encountered in the Wobbler mouse spermatozoa.

Sperm tail abnormalities reported in other animals differ from those found in the Wobbler mouse in that: (1) the plasma membranes are almost always absent or disrupted and (2) degenerating sperm profiles are often associated with obvious clumps of debris. We examined the possibility that abnormal spermatozoa in the Wobbler mouse are degenerating forms as shown in the rat by Cooper \& Hamilton (1977). Only some of the abnormal sperm tails in the Wobbler mouse lacked cytoplasmic membranes and contained vacuolated and dense-granular matrix, both of which probably represent regressive changes. We believe the defects are not artefactual or indicative of dying cells because: (1) control sperm collected at the same time and handled identically showed no alterations; (2) the high incidence of abnormal geometry in apparently well preserved Wobbler spermatozoa and (3) there was no difference in adenylate cyclase content. The increasing degree of abnormality with distal position in the reproductive tract suggests that the sperm tail axoneme in the Wobbler mouse is in some way unstable.

The immotility of spermatozoa with a disordered geometry could account for infertility in the Wobbler mouse but other possibilities could contribute to their reproductive failure: inability to copulate or mount due to weakness, impaired perception of the oestrous female, and dysfunction in ejaculation or other reproductive physiology could also be contributory. We have never observed male Wobbler mice attempting to copulate or leaving vaginal plugs with normal females, although many males seem strong enough to accomplish mounting since hind limbs are usually less affected than forelimbs.

Apparently, only one correlative study has been reported for animals comparing defects in sperm tail axonemes with defects in ciliary axonemes, as was done in Kartagener's Syndrome (Immotile Cilia Syndrome of Afzelius, 1976). Bryan \& Chandler (1978) reported the occurrence of multiple ciliary and sperm tail axoneme defects in a mutant mouse designated, HydrocephalicPolydactyl. The defects reported seem much more severe and variable than we observed in the Wobbler mouse. Another neurological mutant mouse has been reported to have infertility with abnormal spermatozoa but little other information is available (Mullen, Eicher \& Sidman, 1976).

While we are confident that the sperm tail axoneme alterations in the Wobbler mouse are real and significant, we cannot at this stage regard the subtle alterations in efferent duct cilia as linked to the sperm tail defects. The changes observed may represent an artefact even although controls did not reveal any such alterations. Cilia in other locations will have to be examined for structural changes before a systemic axoneme or microtubule defect can be postulated in the Wobbler mouse.

\section{References}

Afzelius, B.A. (1976) A human syndrome caused by immotile cilia. Science, N.Y. 193, 317-319.

Afzelius, B.A., Eliasson, R., Johnsen, O. \& Lindholmer, C. (1975) Lack of dynein arms in immotile human spermatozoa. J. Cell Biol. 66, 225-232.

Andrews, J.M. \& Andrews, R.L. (1976) The comparative pathology of motor neuron diseases. In Amyotrophic Lateral Sclerosis. Recent Research Trends, pp. 181-216. Eds. J. M. Andrews, R. T. Johnson, \& M. A. B. Brazier. Academic Press, New York.

Blom, E. \& Birch-Andersen, A. (1966) The ultrastructure of a new hereditary defect (the "Dag Defect") in the bull sperm tail. In Proc. 5th World Congr. Fert.
Steril., pp. 602-605. Eds B. Westin \& M. Wiqvist. Excerpta Medica, Amsterdam.

Braun, T. (1975) The effect of divalent cations on bovine spermatozoal adenylate cyclase activity. J. Cyclic Nucleotide Res. 1, 271-281.

Bryan, J.H.D. \& Chandler, D.B. (1978) Tracheal ciliary affects in mice homozygous for a recessive pleiotropic mutation Hydrocephalic-Polydactyl. J. Cell Biol. 79, 281a, Abstr.

Chasin, M. \& Harris, D.N. (1976) Inhibitors and activators of cyclic nucleotide phosphodiesterase. Adv. Cyclic Nucleotide Res. 7, 225-264.

Cooper, T.G. \& Hamilton, D.W. (1977) Observations on destruction of spermatozoa in the cauda epidi- 
dymides and proximal vas deferens of non-seasonal male animals. Am. J. Anat. 149, 93-110.

Duchen, L.W., Falconer, D.S. \& Stritch, S.J. (1966) Hereditary progressive neurogenic muscular atrophy in the mouse. J. Physiol., Lond. 183, 53-55.

Duchen, L.W., Stritch, S.J. \& Falconer, D.S. (1968) An hereditary motor neuron disease with progressive denervation of muscle in the mouse: the mutant 'Wobbler'. J. Neurol. Neurosurg. \& Psychiat. 31, 535-542.

Falconer, D.S. (1956) Wobbler (wr). Mouse News Letter $15,23$.

Kartagener, M. (1933) Zur Pathogenese die Bronchiektasien. Beitr. Klin. Tuberk. 83, 489-500.

Kohn, R. (1971) Clinical and pathological findings in an unusual infantile motor neurone disease. $J$. Neurol. Neurosurg. \& Psychiat. 34, 427-431.

Mullen, R.J., Eicher, E.M. \& Sidman, R.L. (1976) Purkinje cell degeneration, a new neurological mutation in the mouse. Proc. natn. Acad. Sci. U.S.A. 73, 208-212.

Olds, P.J. (1971) Effect of the T locus on sperm ultrastructure in the house mouse. J. Anat. 109, 31-37.

Olson, G.E. \& Linck, R.W. (1977) Observations of the structural components of flageller axonemes and central pair microtubules from rat sperm. $J$. Ultrastruct. Res. 61, 21-43.

Pedersen, H. \& Mygind, N. (1976) Absence of axonemal arms in nasal mucosal cilia in Kartagener's syndrome. Nature, Lond. 262, 494-495.

Received 30 May 1979 